(Aus dem physiologischen Institut zu Broslau.)

\title{
Zur Kenntniss des diastatischen Ferments der Lymphe.
}

Von

\section{F. Kähmann.}

Die Untersuchungen von M. Bial haben von Neuem in einwandsfreier Weise gezeigt, dass das Serum des Blutes und der Lynuphe ein saccharificirendes Ferment enthält; sie haben zugleich die Eigenschaften dieses Fermentes genauer kennen gelebrt.

Man konnte die Frage aufwerfen, ob dieses Ferment im Blute des lebenden Thieres circulirt und atus diesem in die Lymphe iibertritt oder ob die saccharificirende Wirkung des Blut- und Lymphserums die Folge postmortaler Veräuderungen des Blut- und Lymphplasmas und der in ilm enthaltenen zelligen Elemente ist. Diese Frage lag nahe, da wir in der Blutgerinnung einen sich erst ausserhalb der Ader entwickelnden fermentativen Prozess kennen und auch das zuckerzerstörende „Ferment" Lépine's sich wenig. stens nach den Versuchen von Arthus erst ausserhalb der Ader bildet.

Es ist nicht leicht dieselbe in einwandfreier Weise zu entscheiden.

Den Nachweis für die Iräexistenz des diastatischen Fermentes im circulirenden Blute suchte Magendie ${ }^{1}$ ) durch intravenöse Injection von Stärkekleister zu führen. Er fand, dass die Jodreaction schon 10 Minuten nach der Injection der Stärke verschwunden war; dagegen liess sich Zucker nachweisen. Die Menge desselben nahm bis zur fünften Stunde zu, dann sank sie allmählich und war nach sieben Stunden völlig verschwunden. Das circulirende Blut des lebenden Thieres besitzt also die Fähigkeit Stärke zu saceharifieiren.

Den Harn fand Magendie in seinen Versuchen zuckerfrei.

1) Compt. rend. XXIII. (1846). 189. 
Aehnliche Injectionen wurden mit wechselndem Resultate in der Folgezeit wiederholt angestellt.

Cl. Bernard) injicirte einem Kaninchen 1 gr löslicher Stärke (nach Béchamp). Dieselbe ging theils als Stärke, theils als Zucker in den Harn über. Der Harn reducirte alkalische Kupferlösung stark und wurde durch Jod blau gefärbt.

Nach Injection von $30 \mathrm{ccm}$ eines filtrirten, stark opalisirenden, also glykogenreichen Leberdecocts in die Venen des Kaninchens fand sich nur einmal Zucker im Harn.

Schiff ${ }^{2}$ ) wies nach, dass bei intravenöser Injection von Dextrin der Harn der Kaninchen, Meerschweinchen und Frösche Zucker, in gewissen Fällen neben diesem auch Dextrin enthält. Nach Injection von Stärkekleister fand er Zucker im Harn und Blut, dagegen fand er keinen Zucker im Harn nach Injection von Granulose und Glykogen.

E. Tiegel ${ }^{3}$ ) spritzte Kaninchen Glykogen, das er unter Zusatz von $0,5 \%$ Kochsalz gelöst hatte, in die Vena jugularis. In einer Reihe von Fällen - die injicirte Menge betrug nur ein Viertel Gramm - beobachtete er keinen Zucker im Harn; nur bei einem ausnahmsweise sehr kräftigen Thier sah er "energischen Diabetes" auftreten.

„Pavy bemerkt, dass nach Injection von Glykogen in die Gefässe des lebenden Thieres das Blut und im Falle der Einführung grösserer Glykogenmengen auch der Harn des Thieres zuckerreich werde.

Tieffenbach erk]ärt die Glykosurie tür eine seltene und nur nach Anwendung beträchtlicher Quantitäten von Glykogen eintretende Folge der Injectionen. Nach Glykogen babe er vergebens im Harn seiner Versuchsthiere gesucht" 4 ).

R. Böhm und F. A. Hoffmann injicirten Katzen 3-10 gr Glykogen in $10 \%$ Lösung im Verlauf einiger Stunden langsam in die Vena jugularis. Der Harn färbte sich allmähich unter Auftreten von Oxybämoglobin zuerst fleischroth, dann purpurroth; er enthielt ,einen stark reducirenden Körper, wahxscheinlich Traubenzucker", ausserdem aber erhebliche Mengen eines Achroodextrins. Letzteres liess sich auch im Blute der Versuchsthiere nachweisen.

Das verschiedene Verhaiten, welches der Harn nach intravenöser Injection von Stärkekleister, Dextrin und Glycogenlösung in den verschiedenen Versuchen zeigte, lässt sich leicht durch die

1) Leçons sur le diabète. Paris 1877. p. 539.

2) Untersuchungen über die Zuckerbildung in der Leber. Würzburg 1859. S. 18 u. Journ. de l'Anat. et de Physiol. 3. (1866). 359.

3) Pflüger's Arch. 6. S. 264.

4) Citirt nach R. Böhm und F. A. Hoffmann. Arch. f. exp. Path. 7 (1877). 489. 
verschiedenen Mengen der eingespritzten Substanz erklären. Wir wissen, dass von dem Zucker, welcher in das Blut direct eingeführt wird, nur dann ein gewisser Procentsatz in den Harn übertritt, wenn die Menge desselben eine bestimmte Grenze überschreitet ${ }^{1}$ ). Ist.nun die Menge der eingesprizten Stärke- oder Glykogenlösung gering, so wird bei der vergleichsweise langsamen Saccharification der Stärke durch Blut, der gebildete Zucker aus dem Blut in die Gewebe übertreten, also kein Zucker im Harn erscheinen. Die Untersuchung des Harnes kann uns dann keinen Aufschluss über die stattgehabte Saccharification geben und auch bei der Untersuchung des Blutes wird sich dieselbe nicht nothwendig durch eine Zunahme des Zuckers zu erkennen geben müssen. Spritzt man grössere Mengen ejn, so finden sich im Harn die Producte der Saccharification, Zucker und Dextrin, wie in den Versuchen von R. Böhm und F. A. Hoffmann. Wir sehen aber gleichzeitig, dass der Harn Hämoglobin enthält. Durch die Injection tritt eine Zerstörung der rothen und vermuthlich auch gewisser farblnser Elemente ein, die sich, wenn sie gering ist, dem Nachweis entzieht, wenn sie stärker wird, durch Hämoglobinurie zu erkennen giebt.

Nchmen wir aber anch an, dass es gelänge jede Alteration des Blutes auszuschliessen, so wïrde das Auftreten von Zucker im Harn oder eine Zunahme des Zuckers im Blut noch nicht für ein Saccharificationsvermögen beweisend sein. Die Zuckerbildung könnte in den Nieren oder anderen Geweben erfolgen.

Wir sehen also, dass man auf diesem Wege nicht in einwandsfreier Weise entscheiden kann, ob das diastatische Ferment in dem normalen circulirenden Blute enthalten ist, wenngleich meiner Ansicht nach nicht zu verkennen ist, dass die mitgetheilten Versuche mit der grössten Wahrscheinlichkeit auf die Anwesenheit eines solchen hindeuten.

Eine andere Methode wäre die, dass man in einen binreiehend grossen Gefässe z. B. in der Jugularis des Pferdes eine gewisse Menge Blut zwischen zwei Ligaturen einschlösse und nun in diesen abgebundenen Theil des Gefässes Stärke- oder Glykogenlösung einspritzte.

1) L. v. Brasol, Wie entleert sich das Blut von einem Ueberschuss an Traubenzucker? Arch. f. Physiol. 1884. S. 211. 
Einen ähnlichen Versuch hat bereits Schiff ${ }^{1}$ ) ausgeführt. „Nous avons àlors injecté une dissolution de glycogène dans une veine d'un lapin au moment de la mort, en emprisonnant la matière injectée et le sang que s'y mêlait au moment de l'injection, entre deux ligatures; et dans ce liquide emprisonné nous avons aussi trouvé du sucre quelques minutes après."

Auch hier könnte man sagen, dass die Stärkeinjection eine Blutveränderung z. B. einen Untergang gewisser Leucocythen bewirkt, welcher die Veranlassung zur Entstehung des saccharificirenden Fermentes wird.

Schiff selbst vertritt noch eine andere Ansicht. Er hatte beobachtet, dass, wenn man nach Injection von Granulose oder Glykogen einen ausgiebigen Aderlass macht, das Blut in zwei Theile theilt und den einen sofort in siedendes Wasser tropfen lässt, den anderen eine Zeit sich selbst uiberlässt, ersterer keinen, letzterer eine grosse Menge von Zucker enthält. Diese Beobachtungen klären sich an einfachsten durch die Langsamkeit, mit welcher nach den Versuchen von Bial das saccharificirende Ferment des Blutes wirkt. Schiff sieht in ihnen den Beweis fuir die postmortale Entstehung des Fermentes. Die Veranlassung zu seiner Entstehung giebt nicht die Gerinnung; denn er findet, dass das Blut vor der Gerinnung eher stärker wirkt als nach derselben, sondern - das Aufhören der Blutbewegung. Aus dem oben erwähnten Versuche und anderen ähnlichen zieht er den Schluss: „C'est l'arrêt du sang ou le ralentissement considérable de son monvement qui sont la canse du développement du ferment diastatique, et l'apparition de ce ferment est le premier symptôme de la mort du sang."

Auch diese Methode hilft also ïber die vorhandenen Schwierigkeiten nicht hinweg. Es gelingt auch so nicht den zwingenden Beweis für das Vorhandensein des diastatischen Fermentes im $\mathrm{kr}$ e i s e nde $\mathrm{n}$ Blute zu erbringen.

Günstiger liegen die Verhältnisse bei der Lymphe. Man kann nachweisen, dass innerhalb der Lymphgefässe, ohne dass die normalen Verbältnisse wesentlich geandert werden, eine Umwandlung von Glykogen in Zucker erfolgt.

1) l. e. 
Auf Veranlassung und mit gütiger Unterstiitzung von Herrn Geheimrath Heidenhain führte ich die folgenden Versuche ans.

Aus einer Fistel des Ductus thoracicus wurde die Lymphe, um weitergehende Umsetzungen $\mathrm{zu}$ verhindern, in Alkohol oder in alkobolischer Löstung von essigsaurem Zink aufgefangen. Meist liess ich zwei mal zehn Cubikcentimeter Lymphe in einen graduirten Cylinder, welcher $40 \mathrm{~cm}$ Alkohol oder $40 \mathrm{cem}$ Alkohol und 21/3 gr essigsaures Zink ${ }^{1}$ ) enthielt, eintropfen. Hierauf liess ich in der Weise, wie dies $\mathrm{Shore}{ }^{2}$ ) in seinen Versuchen mit Pepton that, aus einer Bürette eine $2 \%$ bez. $4 \%$ Lösung von Glykogen in $0,6 \%$ Chlornatrium in ein Lympbgefässs der Pfote einfliessen. Die ersten zehn Cubikcentimeter Lymphe, welche nach Beyinn der Glykogeninjection aus dem Ductus thoracicus ausflossen, wurden nicht weiter untersucht. Hierauf wurden in derselben Weise wie oben meist zwei mal zehn Cnbikcentimeter in Alkohol bez. alkoholischer Lösung von essigsaurem Zink aufgefangen.

Die beiden Proben vor bez. nach der Glykogeninjection wurden vereinigt und in ihnen der Zucker bestimmt.

In den Fällen, wo die Lymphe nur in Alkohol aufigefangen worden war, wurde der Alkohol mitsammt dem Niederschlay in cine Schale geschiittet, die Masse nit einigen Tropfen verdiunnter Essigsäure versetzt, der Alkohol durch wiederholtes Abdampfen unter Zusatz von Wasser entfernt, filtrirt, der Niederschlag auf dem Filter mit heissem Wasser gewaschen, die vereinigten Filtrate eingedampft, genau neutralisirt, anf ein bestimmtes Volumen (meist $30 \mathrm{cem}$ ) aufgefiillt und mit $\mathrm{Kn}$ app'scher Lösung titrirt. Zweckmässiger ist das Verfahren von Abeles.

$$
\text { Versuch I. 27./11. } 91 . \quad \begin{gathered}
\text { Eingctlossen } \\
2 \% \text { Glyk.-Lös. }
\end{gathered}
$$

Lymphe 1. $9 \mathrm{~h} .50$ bis $10 \mathrm{~h} .55$, d. h. in $65 \mathrm{M} .20 \mathrm{com} 0,18 \%$ Zuther

Lymphe II. $11 \mathrm{~h}$. bis $12 \mathrm{~h}, \quad$ d. h. in $60 \mathrm{MI} .16 \mathrm{ecm} 0,22 \%, \quad 7,2 \mathrm{com}$ Lymphe III. $12 \mathrm{~h}$. bis $1 \mathrm{~h} ., \quad$ d. h. in $60 \mathrm{M} .16,5 \mathrm{com} 0,21 \%, \quad 8,0 \mathrm{~cm}$ $11 \mathrm{~h} .45$ Glykogenreaction in der Lymphe zweifelhaft.

12 h. 10

stark.

1) M. Abeles, Ueber ein Verfahren zum knteiweissen des Blutes für die Zuckerbestimmung. Ztschr. f. physiol. Chem. XV. b. S. 495.

2) L. E. Shore, On the fate of peptone in the lymphatic system. The Journ. of Physiology XI. Suppl. N. 1890. S. 583. 


\section{Lymphe I.}

$$
\text { Versuch II. 1./12. } 91 \text {. }
$$

$10 \mathrm{~h} .6$ bis 10 h. 39 , d. h. in 33 M. $10 \mathrm{ccm}$ )

10 h. 39 bis 11 h. 28 , d. h. in 49 M. $10 \mathrm{ccm}\} 20 \mathrm{ccm} 0,09 \%$ Zucker

Lymphe II.

11 h. 35 bis 12 h. 3 , d. h. in 28 M. $10 \mathrm{ccm}$

$6,2 \mathrm{~cm}$

Lymphe III.

$12 \mathrm{~h} .3$ bis $12 \mathrm{~h} .47$, d. h. in 44 M. $10 \mathrm{ccm}$ )

$12 \mathrm{~h} .47$ bis 1 h. 40 , d.h.in 53 M. $10 \mathrm{~cm}\} 20 \mathrm{ccm} 0,15 \%$ Zucker

$6,0 \mathrm{ccm}$

$4,7 \mathrm{ccm}$

Lymphe I.

Versuch III. 4./12. 91 .

$\left.\begin{array}{l}10 \text { h. } 30 \text { bis } 11 \text { h. } 4 \text {, d. h. in } 34 \text { M. } 10 \mathrm{ccm} \\ 11 \text { h. } 4 \text { bis } 11 \text { h. } 38 \text {, d. h. in } 34 \text { M. } 10 \mathrm{ccm}\end{array}\right\} 20 \mathrm{~cm} 0,18 \%$ Zucker

Lymphe II.

11 h. 40 bis 12 h. 14 , d. h. in 34 M. $10 \mathrm{ccm}$

$4,9 \mathrm{~cm}$

Lymphe III.

$12 \mathrm{~h}, 14$ bis $12 \mathrm{~h} .41$, d. h. in 27 M. $10 \mathrm{ccm}$ \} $20 \mathrm{ccm} 0,23 \%$ Zucker $5,8 \mathrm{ccm}$ 12 h. 41 bis 1 h. 24 , d. h. in 43 M. $10 \mathrm{ccm}\} 20 \mathrm{ccm} 0,23 \%$ Zucker $4,6 \mathrm{ccm}$

Versuch IV. 29./1. 92.

Lymphe I. $\quad 9$ h. 50 bis 10 h. 59 , d.h. in 69 M. $25 \mathrm{ccm} 0,12 \%$ Zucker Lymphe II. 12 h. 12 bis 12 h. 28 , d.h. in 16 M. $8 \mathrm{ccm}-\quad 3,2 \mathrm{ccm}$ Lymphe III. 12 h. 30 bis 1 h. 47 , d. h, in 77 M. $25 \mathrm{ccm} 0,22 \%, \quad 9,4 \mathrm{ccm}$

Die Versuche beweisen, dass nach der intralymphatischen Injection von Glykogen ausnahmslos der Prozentgehalt der Lymphe an Zucker steigt.

Zum Vergleich wurden Versuche angestellt, in denen statt der Glykogen-Kochsalz-Lösung nur 0,6\% Kochsalzlösung in das Lymphgefäss einfloss. Hier fand sich niemals eine Zunahme des Zuckers, der prozentische Zuckergehalt der Lymphe blieb der gleiche oder er nahm ab.

Lymphe I.

$$
\text { Versuch } \dot{\text { V. }} \text { 11./12. } 91 .
$$

9 h. 55 bis 10 h. 20 , d. h. in 25 M. $10 \mathrm{ccm}$ )

10 h. 20 bis 10 h. 38 , d. b. in 18 M. $10 \mathrm{ccm}\} 20 \mathrm{~cm} 0,17 \%$ Zucker

$10 \mathrm{~h} .38$ bis 11 h. 15 , d. h. in 37 M. $20 \mathrm{ccm}$

Lymphe II.

11 h. 15 bis 11 h. 35 , d. h. in 20 M. $10 \mathrm{ccm}$

Eingeflossen

$0,6 \%$ ClNa-Lös.

$2,6 \mathrm{ccm}$ 
Lymphe III.

$\left.\begin{array}{l}11 \text { h. } 35 \text { bis } 12 \text { h., } \quad \text { d. h. in } 25 \text { M. } 10 \mathrm{~cm} \\ 12 \text { h. bis } 12 \text { h. } 26 \text {, d. h. in } 26 \text { M. } 10 \mathrm{ccm}\end{array}\right\} 20 \mathrm{ccm} 0,10 \%$ Zucker $\begin{aligned} & 3,6 \mathrm{ccm} \\ & 3,7 \mathrm{ccm}\end{aligned}$

11 h. 35 bis 12 h., $\left.\quad \begin{array}{l}\text { d. h. in } 25 \text { M. } 10 \mathrm{~cm} \\ 12 \text { h. } \quad \text { bis } 12 \text { h. } 26 \text {, d. h. in } 26 \text { M. } 10 \mathrm{~cm}\end{array}\right\} 20 \mathrm{ccm} 0,10 \%$ Zucker $\begin{aligned} & 3,6 \mathrm{ccm} \\ & 3,7 \mathrm{ccm}\end{aligned}$

Eingeflossen

12 h. 31 Fluorescin in das Lymphgefäss der Pfote.

12 h. $35 \quad " \quad$ bei etwas erhöhtem Druck in der Canüle d. D. thor.

\section{Lymphe I.}

Versuch VI. 12./1. 92.

$\left.\begin{array}{r}9 \text { h. } 58 \text { bis } 10 \text { h. } 41 \text {, d. h. in } 43 \text { M. } 10 \mathrm{ccm} \\ 10 \text { h. } 41 \text { bis } 11 \text { h. } 40 \text {, d. h. in } 59 \text { M. } 10 \mathrm{ccm}\end{array}\right\} 20 \mathrm{cem} 0,13 \%$ Zucker

Lymphe II.

1 h. 40 bis 2 h. 2 , d. h. in 22 M. $10 \mathrm{ccm}$

$5,8 \mathrm{ccm}$

Lymphe III.

2 h. 2 bis 2 h. 45 , d. h. in 43 M. $10 \mathrm{~cm}$ \ $20 \mathrm{~cm} 0,12 \%$ Zucker $4,8 \mathrm{ccm}$ 2 k. 45 bis 3 h. 26 , d.h. in 41 M. $10 \mathrm{~cm}\} 20 \mathrm{~cm} 0,12 \%$ Zucker $3,9 \mathrm{ccm}$ 3 h. 30 Fluorescin in d. Lymphgefäss der Pfote.

3 h. 38 , im Chylus.

Lymphe $\mathbf{I}$.

Versuch VII.

9 h. 26 bis 10 h. 24 , d. h. in 58 M. $10 \mathrm{~cm}$ )

$10 \mathrm{~h} .24$ bis $11 \mathrm{~h} .47$, d. h. in 83 M. $10 \mathrm{cem}\} 20 \mathrm{~cm} 0,10 \%$ Zucker

Lymphe II.

12 h. 20 bis 12 h. 59 , d. h. in 38 M. $10 \mathrm{cem}$

$2,9 \mathrm{ccm}$

Lymphe III.

$\left.\begin{array}{l}1 \text { h. bis } 1 \text { h. } 37 \text {, d.h. in } 37 \text { M. } 10 \mathrm{cem} \\ 1 \text { h. } 39 \text { bis } 2 \text { h. } 17 \text {, d.h. in } 38 \text { M. } 10 \mathrm{~cm}\end{array}\right\} 20 \mathrm{ccm} 0,10^{0} / 0$ Zucker $\begin{aligned} & 4,1 \mathrm{ccm} \\ & 6,9 \mathrm{ccm}\end{aligned}$

2 h. 45 Fluorescin in d. Lymphgefäss der Pfote.

2 h. 40 " bei etwas erhöhtem Druck im Chylus; eingeflossen sind $3,5 \mathrm{ccm}$ F'norescinlösung.

Um sicher zu sein, dass die Kochsalzlösung durch das Iymphgefäss in den Ductus thoracicus gelangt war, liess ich am Ende des Versuchs Fluorescin in das Lymphgefäss einfliessen und überzengte mich, ob dasselbe in der Fistel des Ductus thoracicus erschien. Dis Einfliessen der Glykogenlösung in Versuch I bis IV wurde an der Braunfürbung, welche ein Tropfen der aus der Canüle abfliessenden Lymphe nach einer gewissen Zeit bei Zusatz von Jod zeigte, erkannt.

Die Zuckerzunahme nach der Injection von Glykogen kann hiernach nur darauf beruhen, dass in der Lymphe ein diastatisches Ferment euthalten ist, welches die Sacebarification des Glykogens 
164 F. Köhmann: Zur Kenntniss des diastatischen Fermentes der Lymphe.

bewirkt. Es liegt kein Grund vor anzunehmen, dass dasselbe erst unter dem Einfluss der Injection entsteht. Die Circulation in den Lymphbahnen wird nicht wesentlich gestört, das Einfliessen erfolgt langsam, die Lösung des Glykogens in $0,6 \%$ Kochsalz kann als indifferent für die Wand der Lymphgefässe und die geringe Menge der in ihnen enthaltenen farblosen Zellen betrachtet werden.

Diese Versuche gestatten zugleich einen Rijekschluss auf das diastatische Ferment des Blutes.

Das diastatische Ferment der Lymphe kann nur aus dem Blute oder den Geweben herstammen. Ist letzteres der Fall, so 'wird das Ferment durch die Lymphe dem Blute zugeführt. Ein kleiner Theil desselben wird durch den Harn ausgeschieden. Ein Theil bleibt im Blute. Auf ihn wird man die saccharificirende Wirkung desselben beziehen mitssen.

Gelangt das diastatische Ferment in die Lymphe nicht aus den Geweben, sondern ans dem Blute, so sind die obigen Versucbe der directe Beweis dafiur, dass das diastatische Ferment im Plasma des kreisenden Blutes enthalten ist.

Jedenfalls ist die Ansicht von Schiff, dass ,das Erscheinen des diastatischen Fermentes das erste Symptom für das Absterben des Blutes sei ${ }^{\star}$, nicht richtig. 